\title{
Inventário de mosquitos (Diptera: Culicidae) da Unidade de Conservação Ambiental Desterro, Ilha de Santa Catarina, Sul do Brasil
}

\author{
Mariana Reis ${ }^{1}$, Gerson Azulim Müller ${ }^{2,3}$ \& Carlos Brisola Marcondes ${ }^{1}$ \\ ${ }^{1}$ Departamento de Microbiologia e Parasitologia, Centro de Ciências Biológicas, \\ Universidade Federal de Santa Catarina - UFSC, CEP 88040-900, Florianópolis, SC, Brasil \\ ${ }^{2}$ Programa de Pós-graduação em Entomologia, Departamento de Zoologia, \\ Universidade Federal do Paraná - UFPR, CP 19020, CEP 81531-980, Curitiba, PR, Brasil \\ ${ }^{3}$ Autor para correspondência: Gerson Azulim Müller, e-mail: gecoazul@hotmail.com
}

REIS, M., MÜLLER, G.A. \& MARCONDES, C.B. Mosquito survey (Diptera: Culicidae) from Environmental Conservation Unit Desterro, Santa Catarina Island, Southern Brazil. Biota Neotrop. 10(3): http:// www.biotaneotropica.org.br/v10n3/en/abstract?inventory+bn01610032010.

\begin{abstract}
Two thousand and sixty one specimens distributed in 36 species of mosquitoes were collected between August 2007 and May 2008 at the Environmental Conservation Unit Desterro (located in Santa Catarina State, Brazil). The five most abundant species were: Runchomyia reversa (29.74\%), Ochlerotatus scapularis (14.80\%), Wyeomyia pallidoventer (9.51\%), Anopheles cruzii (8.30\%) and Sabethes purpureus $(7.03 \%)$. Ten species of Culicidae are firstly reported to the Santa Catarina State, and of these, six belong to the genus Wyeomyia.

Keywords: Aedes, Anopheles, Atlantic Forest, new records, vectors.
\end{abstract}

REIS, M., MÜLLER, G.A. \& MARCONDES, C.B. Inventário de mosquitos (Diptera: Culicidae) da Unidade de Conservação Ambiental Desterro, Ilha de Santa Catarina, Sul do Brasil. Biota Neotrop. 10(3): http:// www.biotaneotropica.org.br/v10n3/pt/abstract?inventory+bn01610032010.

Resumo: Dois mil e sessenta e um espécimes distribuídos em 36 espécies de mosquitos foram coletadas entre agosto de 2007 e maio de 2008 na Unidade de Conservação Ambiental Desterro (localizada no Estado de Santa Catarina, Brasil). As cinco espécies mais abundantes foram: Runchomyia reversa (29,74\%), Ochlerotatus scapularis $(14,80 \%)$, Wyeomyia pallidoventer $(9,51 \%)$, Anopheles cruzii $(8,30 \%)$ e Sabethes purpureus $(7,03 \%)$. São registradas dez novas espécies de Culicidae para o Estado de Santa Catarina, sendo que dessas, seis pertencentes ao gênero Wyeomyia.

Palavras-chave: Aedes, Anopheles, Mata Atlântica, novos registros, vetores. 


\section{Introdução}

O estudo da fauna de Culicidae é relevante pelo papel que esses insetos desempenham na transmissão de doenças ao homem e a outros vertebrados (e.g. febre amarela). Isso pode ser agravado pelo contato direto entre esses dípteros e a população humana em fragmentos de mata inseridos em ambientes urbanos ou rurais (Navarro-Silva et al. 2004). Áreas de mata preservada em condições semelhantes à situação original em áreas urbanas podem propiciar condições à manutenção de espécies de mosquitos através da oferta de criadouros e fontes de alimentação para os adultos. Dentre os criadouros, podemos destacar os do tipo fitotelmata, os quais são explorados principalmente por espécies com caráter silvestre, além daqueles introduzidos pela atividade antrópica, multiplicando potencialmente a diversidade de recipientes a serem explorados pelos imaturos de culicídeos. O bioma Mata Atlântica apresenta regiões de mata fragmentadas que, muitas vezes, estão inseridas em grandes centros urbanos como é o caso do fragmento pertencente a Unidade de Conservação Ambiental Desterro (UCAD), que abrange uma vasta região de mata em meio a cidade de Florianópolis, Estado de Santa Catarina (CECCA 1997).

Apesar do elevado número de estudos publicados envolvendo a fauna de Culicidae em Mata Atlântica, pouco se sabe a respeito de quais espécies desse grupo se desenvolvem nos fragmentos de mata do Estado de Santa Catarina. Paterno \& Marcondes (2004) em um estudo preliminar na UCAD, observaram 22 espécies de mosquitos, inclusive Anopheles cruzii Dyar \& Knab 1908, responsável pelo surto de malária registrada no estado nas décadas de 1950 e 1960 (Rachou et al. 1973). Dessa forma, o objetivo do presente estudo foi inventariar as espécies de Culicidae que são atraídos durante o dia por humanos na UCAD.

\section{Material e Métodos}

\section{1. Área de estudo}

O material biológico para a realização do estudo foi proveniente da Unidade de Conservação Ambiental Desterro (UCAD), localizada na região Centro-Norte da Ilha de Santa Catarina, Florianópolis, Santa Catarina, Brasil (Figura 1). A sede tem as coordenadas $27^{\circ} 31^{\prime} 50,8^{\prime \prime} \mathrm{S}$ e $48^{\circ} 30^{\prime} 44,3^{\prime \prime}$ O. A UCAD possui 491,5 ha e sua vegetação é do tipo Floresta Ombrófila Densa em quatro estádios de regeneração: capoeirinha (vegetação com até seis anos de desenvolvimento), capoeira (com vegetação se desenvolvendo há aproximadamente 20 anos), capoeirão (vegetação se desenvolvendo por um período de aproximadamente 30 anos) e floresta secundária (área apenas explorada seletivamente) (Bonnet \& Queiroz 2006).

O clima é do tipo Cfa (mesotérmico úmido, com verões quentes e chuvas distribuídas durante o ano) com temperatura média anual de $21,15^{\circ} \mathrm{C}$, média mensal para os meses de verão de $23,46^{\circ} \mathrm{C}$ e para os meses de inverno de $16,75^{\circ} \mathrm{C}$ (Porto Filho 1993). A umidade é alta, em torno de $80 \%$ (CECCA/FNMA 1996), com pluviosidade anual de $1.527 \mathrm{~mm}$, bem distribuída durante todo o ano, não existindo estação seca ou chuvosa (Porto Filho 1993).

\section{Coleta e análise dos dados}

Entre agosto de 2007 e maio de 2008 foram quinzenalmente capturados culicídeos atraídos por humanos através de um tubo de sucção preparado com tubos plásticos transparentes (Marcondes et al. 2007). O operador coletava os mosquitos no momento em que esses pousavam no seu corpo, antes do início do repasto sanguíneo. As coletas duraram dez horas, entre 8:00 e 18:00 horas. Os mosquitos foram mortos em tubo letal contendo acetato de etila e acondicionados em pequenas caixas de papelão para serem levados ao laboratório para a identificação ao nível específico com a utilização das chaves contidas em Lane (1953a, b), Correa \& Ramalho (1956), Consoli \& Lourenço-de-Oliveira (1994) e Forattini (2002). Gêneros e subgêneros de Culicidae foram abreviados de acordo com Reinert (1975). Ochlerotatus foi considerado como gênero de acordo com Reinert (2000).

\section{Resultados e Discussão}

Foram registradas até o momento na UCAD 56 espécies de mosquitos distribuídas em 13 gêneros, sendo que dessas, 36 espécies foram capturadas dentro das amostragens do presente trabalho. Dez espécies foram registradas pela primeira vez no Estado de Santa Catarina, sendo que seis delas pertencem ao gênero Wyeomyia (Tabela 1). Dos 2.061 culicídeos capturados, cinco espécies foram mais representativas: Runchomyia reversa Lane \& Cerqueira 1942 (29,74\%), Ochlerotatus scapularis (Rondani 1848) (14,80\%), Wyeomyia pallidoventer Lutz 1905 (9,51\%), Anopheles cruzii Dyar \& Knab 1908 (8,30\%) e Sabethes purpureus (Theobald 1907) $(7,03 \%)$.

Essas cinco espécies evidenciam um conjunto, que apresenta caráter heterogêneo quanto à exploração de habitats. Ru. reversa Lane \& Cerqueira 1942, a espécie capturada em maior número, é um representante significativo em matas fechadas (Guimarães et al. 2000). A segunda espécie mais abundante, Oc. scapularis (Rondani 1848), destaca-se pela presença em ambientes alterados como matas residuais, nas quais a baixa abundância pode caracterizar o local como sendo de natureza primitiva (Forattini et al. 1995). Wyomyia pallidoventer Lutz 1905, a terceira espécie mais abundante, foi encontrada em bromélias em ambientes antrópicos (Marques et al. 2001) e no interior de fragmentos florestais (Müller \& Marcondes 2006). A quarta espécie, An. cruzii Dyar \& Knab 1908, bem como todas espécies do subgênero Kerteszia, são encontradas em ambientes florestais bem preservados (Dorvillé 1996). Segundo Marcondes \& Paterno (2005), essa espécie apresenta íntima associação de atividade hematofágica com $R u$. reversa Lane \& Cerqueira 1942, devido, provavelmente, a essas duas espécies reagirem de maneira semelhante às condições do ambiente como variação de temperatura e humidade. Sabethes purpureus (Theobald 1907), a última espécie das cinco mais abundantes, é caracterizada por ser uma espécie essencialmente silvestre (Guimarães et al. 2003). Em relação às demais espécies de Culicidae capturadas em número e frequência reduzida, estas também exibem elevada diversidade de habitats.

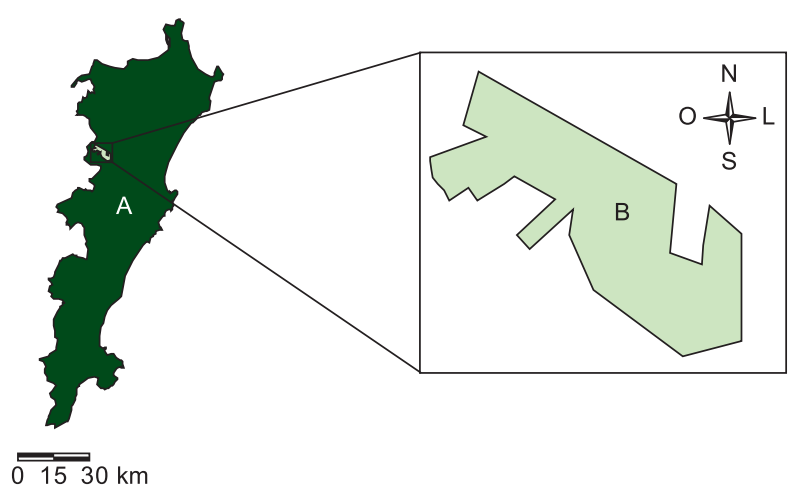

Figura1. Localização da UCAD (B) na região Centro-Norte da Ilha de Santa Catarina (A), SC, Brasil.

Figure 1. Location of UCAD (B) in the North Central part of the Island of Santa Catarina (A), SC, Brazil. 
Tabela 1. Lista de espécies de mosquitos coletadas entre agosto de 2007 e maio de 2008 e de espécies registradas anteriormente na UCAD. Espécies seguidas de $(*)$ representam novos registros para o Estado de Santa Catarina.

Table 1. List of species of mosquitoes collected between August 2007 and May 2008 and the species previously recorded from UCAD. Species followed by (*) represent new records for the State of Santa Catarina.

\begin{tabular}{cccc}
\hline Espécies & $\begin{array}{c}\text { Coletas entre } \\
2007-2008\end{array}$ & $\begin{array}{c}\text { Registros } \\
\text { anteriores }\end{array}$ & $\begin{array}{c}\text { Referências } \\
\text { bibliográficas }\end{array}$ \\
\hline
\end{tabular}

\section{ANOPHELINAE}

Anopheles bambusicolus Komp 1937

An. cruzii Dyar \& Knab 1908

2

171

CULICINAE

AEDINI

Aedes albopictus (Skuse 1894)

Ochlerotatus fulvus (Wiedmann 1828)

Oc. rhyacophilus (Costa Lima 1953)

Oc. scapularis (Rondani 1848)

Oc. serratus s.l. (Theobald 1901)

Oc. terrens (Walker 1856)

Psorophora ferox (Humboldt 1819)

Ps. lutzii (Theobald 1901)

CULICINI

Culex albipes Lutz 1904

Cx. davisi Kumm 1933

Cx. hedys Root 1927

Cx. imitator Theobald 1903

Cx. neglectus Lutz 1904

Cx. pleuristriatus Theobald 1903

SABETHINI

Isostomyia espini Martini 1914*

Limatus durhami Theobald 1901

Li. flavisetosus Oliveira Castro, 1935

Runchomyia cerqueirai Stone 1944*

$R u$. frontosa Theobald 1903*

Ru. humboldti (Lane \& Cerqueira 1942)*

Ru. reversa Lane \& Cerqueira 1942

Ru. theobaldi Lane \& Cerqueira 1934

Sabethes albiprivus Lutz 1903

Sa. aurescens (Lutz 1905)

Sa. melanonymphe (Dyar 1924)

Sa. purpureus (Theobald 1907)

Sa. xyphydes Harbach 1994

Shannoniana fluviatilis (Theobald 1903)

Trichoprosopon digitatum Rondani 1848

Tr. pallidiventer Lutz 1905

Wyeomyia bourrouli Lutz 1905

Wy. chalcocephala Dyar \& Knab 1906*

Wy. complosa Dyar 1928*

Wy. confusa (Lutz 1905)*

Wy. davisi (Lane \& Cerqueira 1942)
7

$-$

1

305

45

$-$

20

1

$\begin{array}{ll}- & \mathrm{X} \\ - & \mathrm{X} \\ - & \mathrm{X} \\ - & \mathrm{X} \\ - & \mathrm{X} \\ - & \mathrm{X}\end{array}$

1

-
X Marcondes et al. (2003), Paterno \& Marcondes (2004)

X Marcondes et al. (2003)

X Paterno \& Marcondes (2004), Marcondes \& Paterno (2005)

X Paterno \& Marcondes (2004)

X Marcondes et al. (2003)

X Paterno \& Marcondes (2004)

Müller \& Marcondes (2006, 2007)

X Müller \& Marcondes (2006, 2007)

X Müller \& Marcondes (2006, 2007)

X Müller \& Marcondes (2006, 2007)

X Müller \& Marcondes (2007)

X Müller \& Marcondes $(2006,2007)$

Paterno \& Marcondes (2004)

X

Paterno \& Marcondes (2004), Marcondes \& Paterno (2005)

$\mathrm{X}$

Marcondes et al. (2003)

$\mathrm{X}$

Paterno \& Marcondes (2004)

X

Marcondes \& Mafra (2003), Marcondes et al. (2003), Paterno \& Marcondes (2004)

$\mathrm{X}$

Marcondes \& Mafra (2003)

Marcondes et al. (2003)

Müller et al. (2008)

Paterno \& Marcondes (2004)

Paterno \& Marcondes (2004)

Marcondes et al. (2003), Paterno \& Marcondes (2004)

Paterno \& Marcondes (2004), Müller \& Marcondes (2006) 
Tabela 1. Continuação...

\begin{tabular}{|c|c|c|c|}
\hline Espécies & $\begin{array}{c}\text { Coletas entre } \\
\text { 2007-2008 } \\
\end{array}$ & $\begin{array}{c}\text { Registros } \\
\text { anteriores }\end{array}$ & $\begin{array}{c}\text { Referências } \\
\text { bibliográficas }\end{array}$ \\
\hline Wy. dyari Lane \& Cerqueira 1942* & 18 & - & \\
\hline Wy. edwardsi (Lane \& Cerqueira 1942) & 1 & - & \\
\hline Wy. fuscipes (Edwards 1922) & 3 & $\mathrm{X}$ & Paterno \& Marcondes (2004) \\
\hline Wy. galvaoi (Correa \& Ramalho 1956) & 30 & $\mathrm{X}$ & Paterno \& Marcondes (2004) \\
\hline Wy. incaudata (Root 1928) & 81 & $\mathrm{X}$ & $\begin{array}{l}\text { Paterno \& Marcondes (2004), Marcondes \& Paterno } \\
\text { (2005), Müller \& Marcondes (2006) }\end{array}$ \\
\hline Wy. lassalli (Bonne-Wepster \& Bonne 1921) & 9 & $\mathrm{X}$ & Müller et al. (2008) \\
\hline Wy. leucostigma Lutz 1904* & 1 & - & \\
\hline Wy. limai Lane \& Cerqueira 1942 & - & $\mathrm{X}$ & Marcondes \& Mafra (2003) \\
\hline Wy. lopesi (Correa \& Ramalho 1956)* & 4 & - & \\
\hline Wy. lutzi (Lima 1930) & - & $\mathrm{X}$ & Marcondes et al. (2003) \\
\hline Wy. palmata (Lane \& Cerqueira 1942) & 3 & $\mathrm{X}$ & Paterno \& Marcondes (2004) \\
\hline Wy. pallidoventer (Theobald 1907) & 196 & $\mathrm{X}$ & $\begin{array}{l}\text { Paterno \& Marcondes (2004), Müller \& Marcondes } \\
\text { (2006) }\end{array}$ \\
\hline Wy. pilicauda (Root 1928) & 79 & $\mathrm{X}$ & $\begin{array}{l}\text { Paterno \& Marcondes (2004), Müller \& Marcondes } \\
\text { (2006) }\end{array}$ \\
\hline Wy. quasilongirostris (Theobald 1907) & - & $\mathrm{X}$ & Marcondes et al. (2003) \\
\hline Wy. splendida Bonne-Wepster \& Bonne 1919 & 2 & $\mathrm{X}$ & Paterno \& Marcondes (2004) \\
\hline Wy. theobaldi (Lane \& Cerqueira 1942) & 129 & $\mathrm{X}$ & Paterno \& Marcondes (2004) \\
\hline Wy. tripartita (Bonne-Webster \& Bonne 1921) & 4 & $X$ & $\begin{array}{l}\text { Marcondes et al. (2003), Müller \& Marcondes } \\
\text { (2007) }\end{array}$ \\
\hline \multicolumn{4}{|l|}{ TOXORHYNCHITINI } \\
\hline $\begin{array}{l}\text { Toxorhynchites bambusicolus } \\
\text { (Lutz \& Neiva 1913) }\end{array}$ & - & $\mathrm{X}$ & Müller et al. (2008) \\
\hline Tx. theobaldi (Dyar \& Knab, 1906) & - & $\mathrm{X}$ & Müller et al. (2008) \\
\hline
\end{tabular}

A presença na área da UCAD, de espécies como Oc. scapularis (Rondani 1848), An. cruzii Dyar \& Knab 1908 e Sa. purpureus (Theobald 1907), que apresentam um comportamento diferenciado quanto às relações com ambiente de floresta primitiva e amplamente modificados pela ação antrópica, demonstrou que embora a urbanização da região tenha englobado a área de mata da UCAD, esta ainda mantém diversidade de condições ambientais, o que tem permitido a manutenção de espécies que possuem caráter silvestre em contato estreito com ambiente alterado.

Entre todas as espécies já registradas na UCAD, várias apresentam competência vetorial em transmitir agentes etiológicos de diversas infecções ao homem in natura em outras áreas e em laboratório, como: An. cruzii Dyar \& Knab 1908 (Branquinho et al. 1997), Ae. albopictus (Skuse 1894) (Miller \& Ballinger 1988), Oc. fulvus (Wiedmann 1828) (Vasconcelos 2003), Oc. scapularis (Rondani 1848) (Forattini 2002), Oc. serratus (Theobald 1901) (Forattini 1965), Ps. ferox (Humboldt 1819) (Lopes et al. 1981), Tr. digitatum Rondani 1848 (Forattini 2002), Wy. bourrouli Lutz 1905 (Labarthe et al. 1998) e Wy. pilicauda (Root 1928) (Lopes \& Sacchetta 1974). Assim, a fauna de culicídeos abrigada pela UCAD pode representar, no futuro, um risco para a população humana presente ao entorno da mesma. Estudos mais aprofundados em busca de mosquitos infectados com arboviroses, helmintoses e protozooses e um monitoramento constante são necessários diante da riqueza de espécies de Culicidae na área e a sua proximidade com habitações humanas.

\section{Agradecimentos}

Ao CNPq pela concessão da bolsa de doutorado para GAM. Ao Sr. Aristides Fernandes (Faculdade de Saúde Pública, Universidade de São Paulo), pela revisão de algumas identificações e ao Sr. Marcos Dupont, pelo auxílio na elaboração da figura 1. Este estudo é parte do Projeto "Internal dynamics of rain forest: specificity of animal-plant interaction" dentro do programa Brasil-Alemanha "Mata Atlântica" (BMBF, proc.- 01LB0205 e CNPq, proc. - 690143/01-0).

\section{Referências Bibliográficas}

BONNET, A. \& QUEIROZ, M.H. 2006. Estratificação vertical de bromélias epifíticas em diferentes estadios sucessionais da Floresta Ombrófila Densa, Ilha de Santa Catarina, Santa Catarina, Brasil. Rev. Bras. Bot., 29(2):217-228.

BRANQUINHO, M.S., MARRELI, M.T., CURADO, I., NATAL, D., BARATA, J.M., TUBAKI, R., CARRERI-BRUNO, G.C., MENEZES, R.T. \& KLOETZEL, J.K. 1997. Infecção de Anopheles (Kerteszia) cruzii por Plasmodium vivax e Plasmodium vivax variante VK247 nos municípios de São Vicente e Juquitibá, São Paulo. Rev. Panam. Salud Publica 2:189-193.

CENTRO DE ESTUDOS CULTURA E CIDADANIA - CECCA, /FNMA. 1996. Uma cidade numa Ilha: relatório sobre os problemas sócioambientais da Ilha de Santa Catarina. Insular, Florianópolis.

CENTRO DE ESTUdOS CULTURA E CIDADANIA - CECCA. 1997. Unidades de Conservação e Áreas protegidas da Ilha de Santa Catarina: caracterização e legislação. Insular, Florianópolis. 
CONSOLI, R.A.G.B. \& LOURENÇO-DE- OLIVEIRA, R. 1994. Principais mosquitos de importância sanitária no Brasil. Fiocruz, Rio de Janeiro.

CORREA, R.R. \& RAMALHO, G.R. 1956. Revisão de Phoniomyia Theobald, 1903 (Diptera, Culicidae, Sabethini). Fol. Clin. Biol. 25:1-176.

Dorvillé, L.F.M. 1996. Mosquitoes as bioindicators of forest degradation in southeastern Brazil, a statistical evaluation of published data in the literature. Stud. Neotrop. Fauna Environ. 31:68-78.

FORATTINI, O.P. 1965. Entomologia médica. Culicini: Culex, Aedes e Psorophora. vol. 2. Faculdade de Higiene Saúde Pública, São Paulo.

FORATTINI, O.P. 2002. Culicidologia Médica. EDSP, São Paulo.

FORATTINI, O.P., KAKITANI, I., MASSAD, E. \& MARUCCI, D. 1995. Studies on mosquitoes (Diptera: Culicidae) and anthropic environment. 9- Synanthropy and epidemiological vector role of Aedes scapularis in South-Eastern Brazil. Rev. Saúde Pública 29:199-207.

GUIMARÃES, A.E., GENTILE, C., LOPES, C.M., SANT'ANNA, A. \& JOVITA, A.M. 2000. Ecología de mosquitos (Diptera: Culicidae) em áreas do Parque Nacional da Serra da Bocaina, Brasil. I- Distribuição por hábitat. Rev Saúde Pública 34:243-250.

GUIMARÃES, A.E., LOPES, C.M., MELLO, R.P. \& ALENCAR, J. 2003. Ecologia de mosquitos (Diptera, Culicidae) em áreas do Parque Nacional do Iguaçu, Brasil: 1-Distribuição por habitat. Cad. Saúde Publica 19:1107-1116.Labarthe, N., Serrão, M.L., Melo, Y.F., Oliveira, S.J. \& Lourenço-de-Oliveira, R. 1998. Potential vectors of Dirofilaria immitis (Leidy 1856) in Itacoatiara oceanic region of Niterói Municipality State of Rio de Janeiro Brazil. Mem. Inst. Oswaldo Cruz 93:425-432.

LANE, J. 1953a. Neotropical Culicidae. vol. 1. EDUSP, São Paulo.

LANE, J. 1953b. Neotropical Culicidae. vol. 2. EDUSP, São Paulo.

LOPES, O.S. \& SACCHETTA, L.A. 1974. Epidemiology of Boracéia Virus in a forested area in São Paulo, Brazil. Amer. J. Epidem. 100:410-413.

LOPES, O.S., SACCHETTA, L.A., FRANCY, D.B., JAKOB, W. \& CALISHER, C.H. 1981. Emergence of a new arbovirus disease in Brazil. III. Isolation of Rocio virus from Psorophora ferox (Humboldt, 1819). Am. J. Epidemiol. 113:122-125.

MARCONDES, C.B. \& MAFRA, H. 2003. Nova técnica para o estudo da fauna de mosquitos (Diptera: Culicidae) em internódios de bambus, com resultados preliminares. Rev. Soc. Brasil. Med. Trop., 36 (6): 763-764.

MARCONDES, C.B. \& PATERNO, U. 2005. Preliminary evidence of association between species of mosquitoes in Atlantic forest of Santa Catarina State, (Diptera: Culicidae). Rev. Bras. Soc. Bra. Med. Trop. 38(1):75-76.

MARCONDES, C.B., ALENCAR, J., BALBINO, V.Q. \& GUIMARÃES, A.E. 2007. Description of three practical and inexpensive devices for the collection of mosquitoes and other small insects. J. Am. Mosq. Control. Assc. 23:84-86.
MARCONDES, C.B., FERNANDES, A., PATERNO, U., MÜLLER, G.A., PINHO, L.C. \& STRUFFALDI, D.V. 2003. New records of mosquitoes from the southern Brazilian states of Santa Catarina and Rio Grande do Sul, with 18 species new for the States (Diptera: Culicidae). Zootaxa 347:1-6.

MARQUES, G.R.A.M., SANTOS, R.L.C. \& FORATTINI, O.P. 2001. Aedes albopictus em bromélias de ambiente antrópico no Estado de São Paulo, Brasil. Rev. Saúde Pública 35(3):243-248.

MILLER, B.R. \& BALLINGER, M.E. 1988. Aedes albopictus mosquitoes intoduced into Brazil: vector competence for yellow fever and dengue viruses. Trans. R. Soc. Trop. Med. Hyg. 82:476-477.

MÜLLER, G.A. \& MARCONDES, C.B. 2006. Bromeliad-associated mosquitoes from Atlantic forest in Santa Catarina Island, southern Brazil (Diptera, Culicidae), with new records for the State of Santa Catarina. Iheringia, Sér. Zool. 96:315-319.

MÜLLER, G.A., KUWABARA, E.F., DUQUE, J.E., NAVARRO-SILVA, M.A. \& MARCONDES, C.B. 2008. New records of mosquito species (Diptera: Culicidae) for Santa Catarina and Paraná (Brazil). Biota Neotrop. 8(4): http://www.biotaneotropica.org.br/v8n4/pt/abstract?shortcommunication+bn01208042008 (último acesso em 07/11/2009).

MÜLLER. G.A. \& MARCONDES, C.B. 2007. Immature mosquitoes (Diptera: Culicidae) on the bromeliad Nidularium innocentii in ombrophilous dense forest of Santa Catarina Island, Florianópolis, Santa Catarina State, southern Brazil. Biotemas 20(2):27-31.

NAVARRO-SILVA, M.A., BARBOSA, A.A. \& CALADO, D. 2004. Atividade de Mansonia spp. Em fragmento florestal na área urbana de Curitiba, Paraná, Brasil. Rev. Brasil. Zool. 21:243-247.

PATERNO, U. \& MARCONDES, C.B. 2004. Mosquitos antropofílicos de atividade matutina em Mata Atlântica em Florianópolis, Santa Catarina, Brasil (Diptera, Culicidae). Rev. Saúde Públ. 38(1):133-135.

PORTO FILHO, E. 1993. Sedimentologia e algumas considerações sobre a bioquímica dos sedimentos do fundo da Lagoa da Conceição, Ilha de Santa Catarina. Dissertação de mestrado, Universidade Federal de Santa Catarina, Florianópolis.

RACHOU, R.G., FERREIRA NETO, J.A. \& MARTINS, C.M. 1973. Observações sobre anofelinos em Santa Catarina. Rev. Soc. Bras. Med. Trop. 7:289-303.

REINERT, J.F. 2000. New classifcation for the composite genus Aedes (Diptera: Culicidae: Aedini), elevation of subgenus Ochlerotatus to generic rank, reclassification of the other subgenera, and notes on certain subgenera and species. J. Am. Mosquito Control Assoc. 16(3):175-188.

REINERT, J.F. 1975. Mosquito generic and subgeneric abbreviations (Diptera: Culicidae). Mosquito System. 7:105-110.

VASCONCELOS, P.F.C. 2003. Yellow fever. Rev. Soc. Bras. Med. Trop. 36(2):275-293 